\title{
Based on the Forwarding Free Riding's Design and Analysis of the Loca- tion Management Method in Wireless Sensor Networks
}

\author{
Min Chen, Ang Li and Lijun Zeng \\ School of Computer Science and Technology, Hunan Institute of Technology, Hengyang, China
}

\begin{abstract}
The paper designs a forwarding Free-Riding-based location management scheme for wireless sensor networks, combining the features of the multi-points routing and the gossip-based broadcast to design the mobile data transmission methods of the objects so that the communication cost can be reduced. The mathematical model of the random Petri network is developed to analyze the behavior of the location management scheme in the WSN so that the method becomes more sophisticated. The experiments show that the forwarding Free-Riding-based location management scheme can reduce the communication cost of the WSN.
\end{abstract}

Keywords: WSN, The Location Management Scheme, Wireless, Network, Sensor, Free-Riding-based.

\section{INTRODUCTION}

The WSN is regarded as one of the important key emerging technologies. The location management technology is one of the most important technologies in the supervising related applications of the WSN. At present, many related applications are applied in the object location management technology. Taking the wild animals tracking as an example, when getting to know the migratory behavior before the coming of the rainstorm, the zoologists can deliver the query to supervise the temperature of the petrel. In order to serve the query, the unlimited sensing network should firstly find out the petrel's located sensors, and then inquire the located sensors transmitted back to the petrel. After receiving the queries obtained from the zoologists, the sensors can transmit the sensing data complying with the requirements of the zoologists and then the data should be transmitted to the zoologists through the WSN and the general Internet $[1,2]$.

According to the above examples, the location-based applications cater to our interests, and the clients adopt the location management scheme to find out the mobile objects. The location query can obtain the required data through the sensor nodes stayed in the mobile objects [3]. A simple method is adopted to realize the location management, and the required sensor networks periodically send the actual locations to the location database. The clients can search for the requested moving objects from the location database of the moving objects with the local location inquiry operation. However, the method can waste a large number of the communication cost in the location for updating and collecting the moving objects because a moving object is easy to change the location. In addition, if the arrival rate of the location inquiry is relatively low, the simple method becomes a low-efficient service for the sensor continuously consumes

*Address correspondence to this author at the School of Computer Science and Technology, Hunan Institute of Technology, Hengyang, China; Tel: 008613607345342;E-mail: 702627@qq.com the energy when updating the database, but it just supplies a few location queries. Therefore, a feasible solving method is that collecting the location data of the moving objects is achieved through the network data processing technology. The above experience shows that the location management in the WSN can be recognized as a new problem of the data management, that is, how to design the data processing technology in the n-network, to effectively maintain the updating of the moving object location, the location query service and the communication cost caused by the minimum target quantity [4-7].

The new location management mechanism is designed by considering the multi-points routing and the features of the Gossip-based broadcast. The multi-points routing can be naturally matched with the traditional tree structure and the Forwarding structure, while the Gossip-based broadcast can conduct the Free-rider-based data deletion. The so-called Free-rider-based data deletion means that another node which has the stale location data and whose location is next to the registering node can obtain that the users have been registered by other nodes through the features of the Gossipbased broadcast when a node is conducting the location updating of the moving objects. Therefore, the stale data can be deleted by itself. In this way, the wireless sensor system is unnecessary to spend the extra communication cost to delete the stale data. The features of the above two hardwares should be brought into the design of the location management mechanism, that is, the mechanism belongs to the new mechanism. Aiming to the location management scheme designed for the WSN, a large amount of the communication cost can be reduced through the experimental results [8-10].

The Forwarding Free-rider-based location management scheme is proposed in the paper, and the multi-points routing and the features of the Gossip-based broadcast can make the location management scheme of the WSN more efficient. The communication cost of the moving object can be reduced on the basis of the maximal behavior of the Free-riderbased data deletion with the use of the special communication features of the sensors, that is, the Gossip property. The 
outdated location data in the tree data structure of the moving objects should be deleted. The locations of the moving objects are very closing for the locations of the moving objects have the geographic continuity. Therefore, the node can automatically delete the original location data through the Gossip property without costing the extra communication cost [11-14].

In order to optimize the performance of the Forwarding Free-rider-based location management scheme, a mathematical analysis model based on the Markov Chain is developed by adopting the Stochastic Petri Net to estimate the communication cost. The estimated results of their efficiency can get to know the advantages of the location management scheme of the multi-points routing and the features of the Gossip-based broadcast and its simulated results show that the Forwarding Free-rider-based location management scheme is superior to other location management schemes [15].

Other chapters in the paper are as follows: The Chapter Two describes the system model, the Chapter Three is the Forwarding Free-rider-based location management scheme, the Chapter Four is the model of the efficiency estimation, the Chapter Five is the experimental results and the Chapter Six is the conclusion.

\section{THE SYSTEM MODE}

The Fig. (1) shows the moving management schemes in the WSN. A large number of sensors are often deployed in the observation sites by the WSN. Each sensor has the ability of the perception, the calculation and the communication, having the location in the WSN. When there is a location request of a moving object, the WSN can adopt the existing localization techniques to estimate the location of the moving objects, and deliver the data between the two nodes (such as the location query).

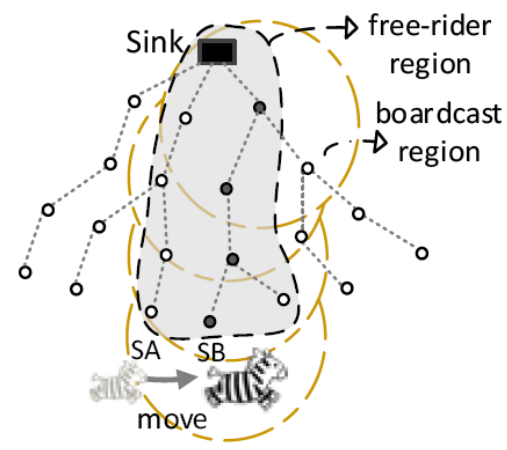

Fig. (1). The model of the WSN.

The sensor nodes adopt the broadcast protocol to transmit the data to the neighboring nodes in the WSN. Therefore, the sensors have the communicating features, that is, the Gossip property. When there is a sensing node broadcasting, all neighboring nodes can hear the transmitted information (such as the grey area which is defined as the free-rider region). Even if they are not the target nodes or the relay nodes, they can also be represented by the orange circle in the Fig. (1). The Gossip radius can adjust the transmitting power of a sensor node to make it more moving. In order to increase its flexibility, a tree-structure WSN is deployed to transmit the data and has the geographic multi-hop routing protocol. Each node is assumed to maintain the node ID and the location in its sub-tree for knowing the relationship of the neighboring nodes in the space.

\section{THE FORWARDING FREE-RIDER-BASED LOCA- TION MANAGEMENT SCHEME}

The main designing spirit of the Forwarding Free-riderbased location management schemes is to adopt the GossipProperty to automatically delete the original location data (it is called the Free-rider-based register procedure) and combine the Forwarding structure for reducing the communication cost. The Free-rider-based register procedure can reduce the probability of deleting the packets of the outdated location data. The location management scheme can be divided into two operating mechanisms and a procedure, the updating of the object location, the location query service and the Free-rider-based register procedure. Its operating method can be described as follows.

The updating of the object location mechanism:

When an object (O1) moves from a sensor (SA) to another sensor (SB), the $\mathrm{O} 1$ can conduct the location updating to the SB and the transmit the Forwarding length (K). The SB can record the location data of the $\mathrm{O} 1$ and its Forwarding length should be added with a $(\mathrm{K}+1)$.

If the SB finds that the Forwarding length of the $\mathrm{O} 1$ is larger than the maximal Forwarding length (KMAX) set in the system, the SB will initiate the Free-rider-based register procedure to conduct the location updating of the $\mathrm{O} 1$ to the Sink, and the Forwarding length of the $\mathrm{O} 1$ becomes 0 and the Anchor of the O1 is set as SB. At this time, the Sink will record the $\mathrm{O} 1$ stored in the $\mathrm{SB}$.

If the $\mathrm{SB}$ finds that the Forwarding length of the $\mathrm{O} 1$ is smaller than the KMAX, the SB will establish the forwarding link to the SA, and the SA will record the O1 stored in the SB.

The Free-rider-based register procedure:

When the SB initiates the location query service to conduct the location updating of the $\mathrm{O} 1$ to the Sink, its Anchor of the $\mathrm{O} 1$ is $\mathrm{SO}$ and the $\mathrm{SB}$ is at the $\mathrm{n}$ stage of the treestructure. The SB can transmit the $\mathrm{O} 1$ register packets according to the previous stage sensor (SBn-1) of the SB in the tree structure. The $\mathrm{O} 1$ register packets include the node SB in the O1, the O1 Anchor SO, the ID in the reception terminal SBn- 1 and the transmitting terminal SB and the location coordinates.

If any sensor (SOn-1) adopts the Gossip-Property to hear the $\mathrm{O} 1$ register packets transmitted by the SBn-1, the SOn should check whether it has the O1 information. If so, the SOn-1 will automatically delete the $\mathrm{O} 1$ data and check whether there is Gossip-Property available in the next stage sensor (SOn) of the SOn-1 to hear the O1 register packets.

The SOn-1 adopts the location coordinates SBn-1 and SB to calculate whether the distance of the SB and the $\mathrm{S} O \mathrm{On}$ is larger than the Gossip radius. If so, the SOn can hear the O1 register packets and conduct the last step. If no, the SOn-1 should send the register packets to the SOn for deleting the O1 data in the SOn. 
The SBn-1 should transmit the O1 register packets to the last-layer tree structure (SBn-2), and directly to the Sink.

The location query service mechanism:

When there is a $\mathrm{O} 1$ location query, the Sink can transmit the inquiry packets to the SO according to the node SO recorded by the $\mathrm{O} 1$.

If the $\mathrm{SO}$ receives the inquiry packets and finds out that the $\mathrm{O} 1$ is within the sensing range itself, the $\mathrm{SO}$ is the Anchor and the Forwarding length is 0 . The SO transmits the O1 data required by the inquiry packets to the Sink.

If the $\mathrm{SO}$ receives the inquiry packets and the $\mathrm{O} 1$ is within the SA range, the $\mathrm{SO}$ is the Anchor and the Forwarding length is not 0 . The $\mathrm{SO}$ will transmit the inquiry packets to the SA along with thee Forwarding. The SA will conduct the confirmation of the last step until the $\mathrm{O} 1$ is within the sensing range itself.

\section{THE EFFICIENCY ESTIMATION MODEL}

A mathematical model is developed in this chapter and the random Petri Internet (SPN) is used as a tool to analyze the proposed scheme.

\subsection{The Cost Model}

The communication performance of the transmission cost of the whole energy is used as the indicator, and the data in each unit time is the estimation and analysis of the location management scheme so that the packet transmission is the most power-consumed operation of the sensors. Therefore, the schemes used to estimate the location management in the cost model include two component cost, the average cost $\left(C_{\text {update }}\right)$ of the location updating service and the average $\operatorname{cost}\left(C_{\text {query }}\right)$ of the defined objects. The $C_{\text {total }}$ is defined to offer the operating cost of the location updating and the inquiry of the moving object location, as shown in the following.

$$
C_{\text {total }}=\lambda \times C_{\text {update }}+\mu \times C_{\text {query }}
$$

in which $\lambda$ and $\mu$ are the rate of the location updating and the rate of the location inquiry.

\subsection{The Cost Model}

The Fig. (1) represents the random Petri Internet in the Forwarding Free-rider-based location management scheme, and the SPN model simulates the behavior of the moving object and the inquiry arrival. Therefore, the communication cost can be calculated through the model. The Table 1 represents the Place and the significance of the Transition in the SPN model. In addition, the mark(p) equation represents the marks (token) numbers of the place $\mathrm{p}$. The SPN model in the Forwarding Free-rider-based location management scheme can be divided into three parts in terms of the operating behavior in the scheme, the Location update part, the Chain maintenance part and the Location query part. Their detailed descriptions are as follows:

\subsubsection{The Location Update Part}

When an object moves to a new sensor, it should be simulated to a mark moving from Move In and O Move, in which the rate of producing the marks by the Move In is $\lambda$.

Table 1. The definition of the Place and the Transition in the SPN model.

\begin{tabular}{|c|c|}
\hline Names & Definitions \\
\hline O Move & $\begin{array}{l}\operatorname{mark}(\mathrm{O} \text { Move })=1 \text { represents that an object moves to } \\
\text { another sensor }\end{array}$ \\
\hline O Level & $\begin{array}{l}\operatorname{mark}(\mathrm{O} \text { Level}) \text { represents the class number of the } \\
\text { objects }\end{array}$ \\
\hline Up & $\begin{array}{l}\text { The class location relationship between the new and } \\
\text { old sensors is down }\end{array}$ \\
\hline Update Chain & $\begin{array}{l}\text { The new chain structure is caused by the object } \\
\text { movement }\end{array}$ \\
\hline Forward & The object moves forward (moving to a new node) \\
\hline Back & $\begin{array}{l}\text { The object moves backward (moving to the last } \\
\text { node) }\end{array}$ \\
\hline K Value & The length of the Forwarding chain \\
\hline Anchor Level & $\begin{array}{l}\text { mark(Anchor Level) represents the class number of } \\
\text { the objects }\end{array}$ \\
\hline Query & $\operatorname{mark}($ Query $)=1$ represents a location inquiry \\
\hline Move In & The object movement, and its rate is $\lambda$. \\
\hline O Up & $\begin{array}{l}\text { The class location relationship between the new and } \\
\text { old sensors is up, and the possibility is } \mathrm{P} \text { up. }\end{array}$ \\
\hline O Down & $\begin{array}{l}\text { The class location relationship between the new and } \\
\text { old sensors is down, and the possibility is P down. }\end{array}$ \\
\hline O Equal & $\begin{array}{l}\text { The class location relationship between the new and } \\
\text { old sensors is translation, and the possibility is P } \\
\text { same. }\end{array}$ \\
\hline Decrease Level & The class number is reduced a layer \\
\hline Move Forward & $\begin{array}{l}\text { The object moves forward, and the possibility is } P \\
\text { fwd }\end{array}$ \\
\hline Move Back & $\begin{array}{l}\text { The object moves backward, and the possibility is } \\
P_{\text {back }}\end{array}$ \\
\hline Add pointer & $\begin{array}{l}\text { The length of the Forwarding chain adds one, and } \\
\text { the rate is } r_{\text {add }} \text {. }\end{array}$ \\
\hline Remove Pointer & $\begin{array}{l}\text { The length of the Forwarding chain minuses one, and } \\
\text { the rate is } r_{r e m} \text {. }\end{array}$ \\
\hline Clear Chain & $\begin{array}{l}\text { The Forwarding chain should be updated, and the } \\
\text { rate is } r_{c c} \text {. }\end{array}$ \\
\hline Query In & The causing of the location query, and the rate is $\mu$. \\
\hline Query Processing & After the location inquiry is served, its rate is prow. \\
\hline
\end{tabular}




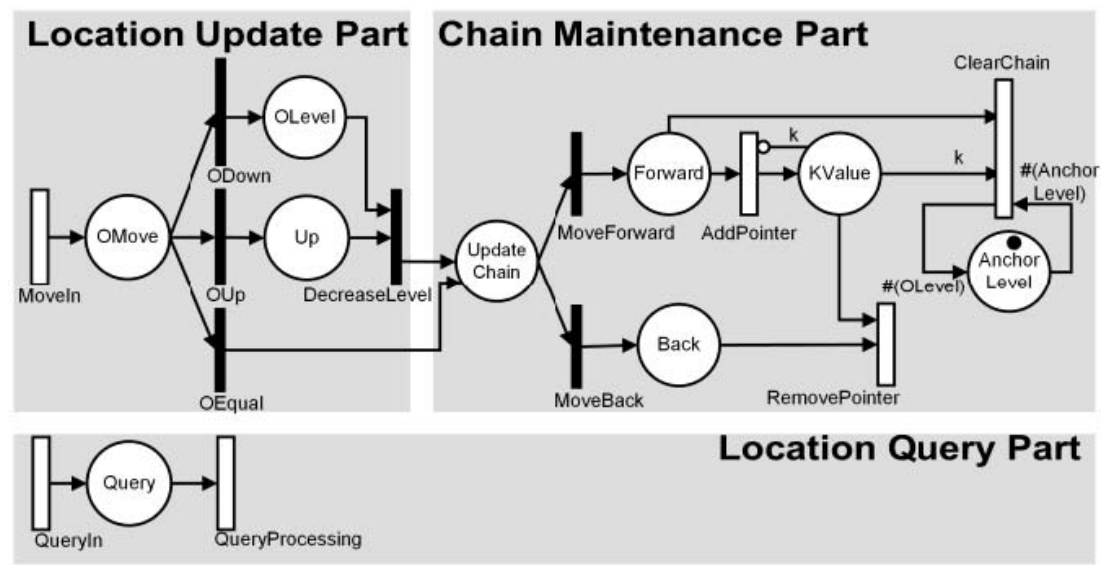

Fig. (2). The SPN model in the Forwarding Free-rider-based location management scheme.

The class location relationship between the new sensor and the last sensor has three possibilities, down, up and translation, which are simulated as O Down, O Up and $\mathrm{O}$ Equal by the SPN, and the possibility of not changing the class number are $\mathrm{P}$ down, $\mathrm{P}$ up and $\mathrm{P} \mathrm{S}$ same.

If the class location relationship is down, a mark should be placed in the O Level by the O Down, in which the mark number of the $\mathrm{O}$ Level represents the class number of the new sensors. The Forwarding operation should be initiated and the mark which is simulated by the $\mathrm{O}$ Down should be placed in the Update Chain.

If the class location relationship is up, a mark in the $\mathrm{O}$ Down should outflow to the Decrease Level. It represents that the class number should be reduced by a layer. The Forwarding operation should be initiated and the mark which is simulated by the Decrease Level should be placed in the Update Chain.

If the class location relationship is translation, the class number is not changed. The Forwarding operation should be initiated and the mark which is simulated by the $\mathrm{O}$ Equal should be placed in the Update Chain.

\subsubsection{The Chain Maintenance Part}

After the object moves forward, it can conduct the Forwarding chain maintenance and be simulated as a mark in the Update Chain. A mark can move to a new node or move to the last node backward. In other words, the length of the Forwarding should add 1 or minus 1 . The SPN model adopts the Move Forward and the Move Back to distinguish the situation and its possibility is $P_{f w d}$ and $P_{b a c k}$ respectively.

If the object moves forward, the Update Chain will outflow a mark and the Forward places a mark.

If the length of the Forwarding is smaller than the maximal Forwarding length $(\mathrm{K})$, the Forward will outflow a mark and the Add Pointer will produce a mark which is placed in the $K_{\text {Value }}$. The mark number of the $K_{\text {Value }}$ represents the Forwarding length.

If the length of the Forwarding is larger than the maximal Forwarding length $(\mathrm{K})$, it represents the updating Forwarding and the initiation of the Free-rider-based register procedure.
The Forward will outflow a mark which is in the Clear Chain, and the marks of the $K_{\text {Value }}$ will outflow totally.

When the Forwarding chain is updated and the Freerider-based register procedure is initiated, the location of Anchor will be updated, and it is simulated as the Anchor Level to delete the marks in the state itself and place the marks in the $O_{\text {Level }}$.

After the object moves backward, the Update Chain can outflow a mark which is placed in the Back. The Forwarding chain will be deleted and the length of the Forwarding will minus. It should be respectively simulated as the Back and the $K_{\text {Value }}$ to outflow a mark.

\subsubsection{The Location Query Part}

After the location query is produced, a mark is simulated from the Query In to the Query, and the Query In produces the rate of the mark $\mu$.

When the location query finds out the object and then should be transmitted back to the sink, a mark is simulated from the Query to the Query Processing and the processing rate is $r_{\text {pro }}$.

\subsection{The Parameterization}

The $P_{\text {down }}$, the $\mathrm{P}$ up and the PS same can be obtained from the number of the class nodes in the tree network, and it can be estimated by the number of the nodes from the upper class to the lower class. Take the $P$ up as an example, the present class is Lo and its value is the possibility that all probable nodes may exist in the last layer. In the same way, $P_{\text {down }}$ is the possibility that all probable nodes may exist in the next layer, and the $P_{\text {same }}$ is the possibility that all probable nodes may exist in the current layer. The following equation is as follows:

$$
\begin{aligned}
& P_{\text {down }}=\frac{\operatorname{level}(\operatorname{Lo}+1)}{\operatorname{level}(\operatorname{Lo}+1)+\operatorname{level}(\operatorname{Lo}+1)+\operatorname{level}(\operatorname{Lo}+1)} \\
& P_{u p}=\frac{\operatorname{level}(\operatorname{Lo}-1)}{\operatorname{level}(\operatorname{Lo}+1)+\operatorname{level}(\operatorname{Lo}+1)+\operatorname{level}(\operatorname{Lo}+1)}
\end{aligned}
$$




$$
P_{\text {same }}=\frac{\operatorname{level}(\operatorname{Lo})}{\operatorname{level}(\operatorname{Lo}+1)+\operatorname{level}(\operatorname{Lo}+1)+\operatorname{level}(\operatorname{Lo}+1)}
$$

in which level(Lo) is the total node numbers in the Lo stage. The area covered by the Lo layer in the sensing range can be obtained by adding the sensing range and the numbers of the sensors. The distance $\left(R_{L o}\right)$ from the sink to the Lo and the distance $\left(R_{L o-1}\right)$ from the sink to the Lo-1 are as follows:

$\operatorname{level}(L o)=\frac{R_{L o}^{2}-R_{L o-1}^{2}}{R^{2}} \times N$

in which the $P_{f w d}$ is the probability when the object moves to the non-former position node. The math is used to represent the probability when all neighboring nodes cannot appear in the previous nodes, which can be shown by the following formula. All neighboring nodes number $N_{\text {sur }}$ (the node numbers are within the sensor 1hop) in the following experiments is 9 .

$P_{f w d}=\left\{\begin{array}{l}1, \text { ifMark }(\text { KValue })=0 \\ \frac{N_{\text {sur }}-1}{N_{\text {sur }}}, \text { otherwise }\end{array}\right.$

in which $P_{\text {back }}$ is the probability when the object moves to the former position node. The following formula represents the probability when all neighboring nodes appear in the previous nodes.

$P_{\text {back }}=\left\{\begin{array}{l}0, \text { ifMark }(\text { KValue })=0 \\ \frac{1}{N_{\text {sur }}} \quad, \text { otherwise }\end{array}\right.$

The rate of the Query Processing is $r_{p r o}$, it represents that the query packets will pass $n_{q}$ hop. In other words, $n_{q}$ query packets should be transmitted each second, in which $n_{q}$ is the nodes from the sink to the Anchor along with the Forwarding chain to the nodes in the object, as shown in the following formula.

$$
r_{\text {pro }}=\frac{1}{n_{q}}=\frac{1}{\operatorname{Mark}(\text { Anchor Level })+\operatorname{Mark}(\text { KValue })}
$$

The rate of the Add pointer and the Remove Pointer is $r_{\text {add }}$ and the $r_{\text {rem }}$ for establishing the chain to transmit a register packet and a Back to the former node. Therefore, two hops are just required and can be represented by the following formula:

$r_{\text {add }}=r_{\text {rem }}=\frac{1}{2}$

The average cost ( $C_{\text {update }}$ ) of the position updating service is the cost of adding or deleting the Forwarding chain and the cost of updating the Forwarding chain. The cost of updating the Forwarding chain can be divided into two types, the cost $\left(C_{\text {res }}\right.$ ) of updating the position to the sink and the cost $\left(C_{D e l}\right)$ of deleting the old data, which can be shown as follows:

$$
\begin{aligned}
C_{\text {update }} & =C_{p t r}+\left(C_{\text {res }}+C_{\text {del }}\right) \\
= & P_{p t r} \times 2 \tau+P_{\text {chain }} \times\left(c_{\text {res }}+c_{\text {del }}\right)
\end{aligned}
$$

in which the $P_{p t r}$ is the probability of adding or deleting the Forwarding chain. It can be obtained by the Forward and the Back in the SPN. $P_{\text {chain }}$ is the probability of updating the Forwarding chain and it can be obtained by the $K_{\text {Value }}$ in the SPN. $C_{r e s}$ represents the registering cost by averagely updating objects data to the sink, and $C_{D e l}$ represents the solving registering cost by averagely deleting the outdated data.

$C_{r e s}=\operatorname{Mark}($ OLevel $) \times \tau$

$C_{\text {del }}$ represents the solving registering cost by deleting the outdated data from the Anchor to the sink and the Forwarding chain. The Free-rider-based register procedure in the scheme can reduce the times (NFR) of transmitting the solving register packets, in which FR(i) represents whether the nodes with the solving-willing register in the $i$ stage are the nodes of the Free Rider solving register. $\mathrm{n}$ is the stage number of the nodes in the current object and $d(i)$ is the distance between the Anchor and the father nodes in the $i$ layer of the nodes in the object. If the distance is lower than the maximal distance of the Gossip, the Gossip-Property can be adopted to hear the register packets. If no, the GossipProperty cannot be adopted.

$$
\begin{aligned}
& C_{r e s}=\left(\operatorname{Mark}(\text { Anchor Level })+\operatorname{Mark}(\text { KValue })-N_{F R}\right) \times \tau \\
& N_{F R}=\sum_{i=0}^{n} F R(i), \text { where FR }(i)= \begin{cases}1, \text { if }\left(d(i)<R_{\text {gos }}\right) \\
0, & \text {, otherwise }\end{cases}
\end{aligned}
$$

The average cost ( $C_{\text {query }}$ ) in the defined objects should be found out and then the communication cost of each query from the sink to the Anchor and then to the sensors in the objects, can be calculated as follows.

$$
C_{\text {query }}=P_{q} \times(\operatorname{Mark}(\text { Anchor Level })+\operatorname{Mark}(\text { KValue })) \times \tau
$$

\section{THE EXPERIMENTAL RESULTS}

The experimental results are analyzed in this chapter, through the SPN analysis model calculation. The Table 2 shows the parameters and settings of the experimental system. The chapter rebuilds the pointer forwarding scheme which should be complied with the WSN as the comparing objects. Compared with the Forwarding Free-rider-based position management scheme, it has no Free-rider-based 
register procedure. In other words, the Forwarding scheme should transmit the solving register packets to each node with the outdated data.

Table 2. The definition of the Place and the Transition in the SPN model.

\begin{tabular}{|l|l|}
\hline \multicolumn{1}{|c|}{ Names } & \multicolumn{1}{c|}{ Definitions } \\
\hline \hline Sensing range $(\mathrm{R})$ & $500 \quad 500$ square meters \\
\hline the number of the sensors $(\mathrm{N})$ & 1000 \\
\hline communicating range & 150 square meters \\
\hline the maximal class number & 30 \\
\hline The moving speed of the object $(\lambda)$ & {$[0.1 \sim 1.0]$ each/seconds } \\
\hline The query arrival speed $(\mu)$ & 0.1 each/seconds \\
\hline $\begin{array}{l}\text { The ratio between the object movement } \\
\text { and the query }(\mathrm{QMR}=\lambda / \mu)\end{array}$ & 0.5 \\
\hline The cost $(\tau)$ by the data transmits a hop & 1 \\
\hline
\end{tabular}

\subsection{The Comparison of the Efficiency}

The Fig. (2) shows the Forwarding Free-rider-based position management scheme's and the Forwarding scheme' $\mathrm{s}$ influences on the total communication cost in different QMR. When the moving times are higher (the QMR is larger), the cost of the Forwarding Free-rider-based position management scheme is lower than Forwarding scheme's for the Forwarding Free-rider-based position management scheme adopts the Gossip-Property to delete the outdated data. Compared with the Forwarding scheme, the Forwarding Free-rider-based position management scheme can reduce the deleted packets during the process of transmitting the outdated data. Therefore, when the rate of the object movement is higher, the communication cost can be largely reduced.

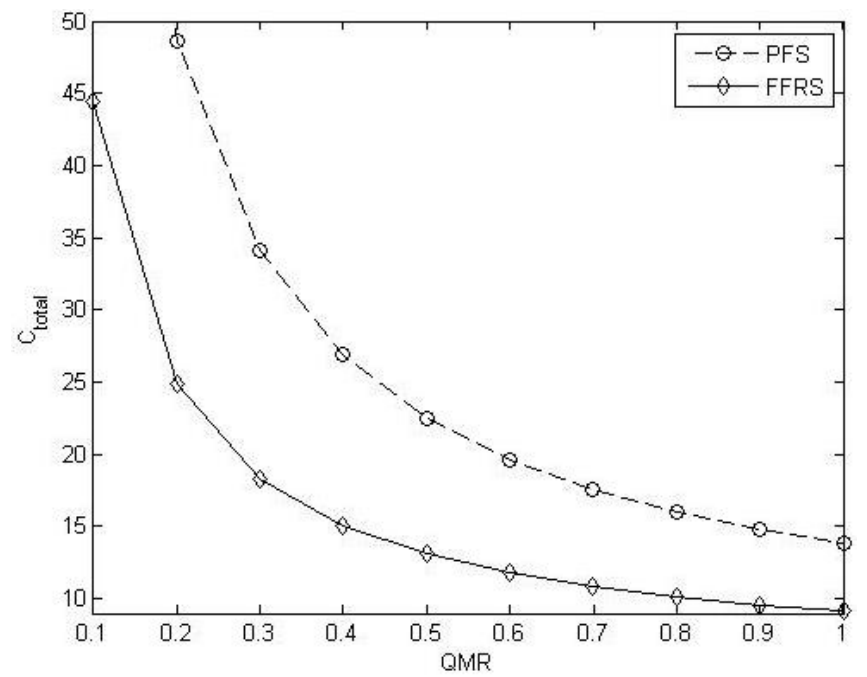

Fig. (2). The QMR's influence on the Forwarding Free-rider-based position management scheme and the PFS total communication cost

\subsection{The Analysis of the Sensibility}

The Fig. (3) shows that the different communication distance in the Forwarding Free-rider-based position management scheme and the Forwarding scheme influences the total communication cost difference in the two schemes. If the Forwarding Free-rider-based position management scheme can improve the high cost with the increase of the communication distance, the Forwarding Free-rider-based position management scheme adopts the Gossip-Property to delete the outdated data. When the communication distance is larger, there are more nodes available, which can adopt the Gossip-Property to delete the outdated data. In other words, the times of transmitting the solving register packet can be reduced. In the same way, the cost of the Forwarding scheme is not influenced by the communication distance. In other words, the times of transmitting the solving register packet cannot be reduced with the reduction of the communication distance. Therefore, the larger the sensor communication distance is, the smaller the communication cost is.

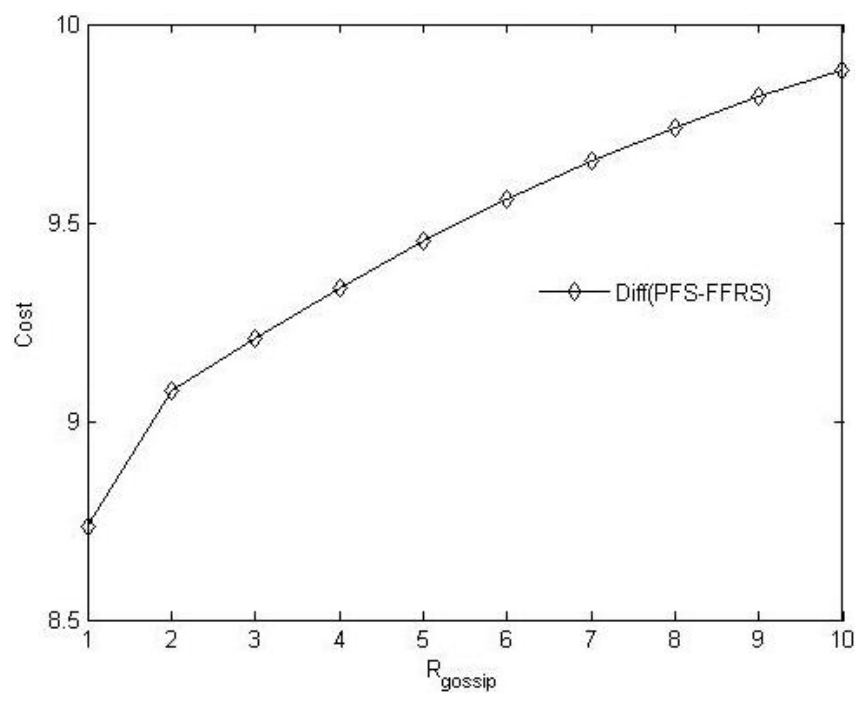

Fig. (3). The communication distance's influence on the Forwarding Free-rider-based position management scheme and the PFS total communication cost

\section{CONCLUSION}

This paper designs a Forwarding Free-rider-based position management scheme by combining multi-points routing and the features of the Gossip broadcast so that the communication cost can be reduced. Then it develops a SPN mathematical model to analyze the behavior of the Forwarding Free-rider-based position management scheme in the WSN so that the efficiency of the Forwarding Free-rider-based position management scheme can be analyzed.

\section{CONFLICT OF INTEREST}

The authors confirm that this article content has no conflict of interest.

\section{ACKNOWLEDGEMENTS}

Declared none. 


\section{REFERENCES}

[1] R.V. Kulkarni, A. Forster, and G.K. Venayagamoorthy, "Computational Intelligence in Wireless Sensor Networks: A Survey, " IEEE Communications Surveys \& Tutorials, vol. 13, no. 1, pp. 68-96, 2011.

[2] C.C. Chen, and C.H. Liao, "Model-based Object Tracking in Wireless Sensor Networks," Wireless Networks, vol. 17, no. 2, pp. 549$565,2011$.

[3] C. Hirel, B. Tuffin, and T.S. Kishor, " SPNP: Stochastic petri nets. version 6.0," Springer Berlin, Heidelberg, vol. 1786, pp. 354-357. 2000.

[4] Y. Li, and I. R. Chen, "Design and performance analysis of mobility management schemes based on pointer forwarding for wireless mesh networks," IEEE Transactions on Mobile Computing, vol. 10, no. 3, pp. 349-361, 2011.

[5] V.S. Bhadauria, S. Sharma, and R. Patel, "Reducing overhead in movement based dynamic location management scheme for cellular networks," Telecommunication Systems, vol. 57, no 1, pp. 1-11, 2014.

[6] B. Liu, L. Zhang, and D.F. Josep, "On the security of a privacypreserving key management scheme for location based services in VANETs," Lecture Notes in Computer Science (including subseries Lecture Notes in Artificial Intelligence and Lecture Notes in Bioinformatics), vol. 8352, pp. 323-335, 2014.

[7] R. Vaid, and V. Katiyar, "VLKM: virtual location-based key management scheme for Wireless Sensor Networks," In: International Conference on Parallel, Distributed and Grid Computing (PDGC), pp. 53-61, 2014.

[8] Nath P, Kumar C, "Mobility Management Scheme for Fixed Mobility Pattern Mobile Users in IPv4 Networks," Computer Journal, vol. 57 , no. 12 , pp. 131-49, Dec. 2014.

[9] K. Huang, D. Li, and Y. Sun, "CRMS: a centralized replication management scheme for cloud storage system," IEEE/CIC International Conference on Communications in China (ICCC), pp. 344-8, 2014.

[10] K. Huang, D. Li, and Y. Sun, "CRMS: A centralized replication management scheme for cloud storage system," IEEE/CIC International Conference on Communications in China, ICCC 2014, pp. 344-348, 2015.

[11] M. Almulla, Y. Wang, A. Boukerche, and Z. Zhang, "Design of a Fast Location-Based Handoff Scheme for IEEE 802.11 Vehicular Networks," IEEE Transactions on Vehicular Technology, vol. 63, no. 8, pp. 3853-3866, 2014.

[12] A. Chandra, and P. Das, "Location Management in Cellular Mobile Networks," IEEE Potentials, vol. 33, no. 1, pp. 37-44, 2014.

[13] S. Shiokawa, and D. Chen, "Location based clustering scheme considering node mobility in wireless sensor networks," In: Sixth International Conference on Ubiquitous and Future Networks (ICUFN), pp. 149-53, 2014.

[14] A.N. Letchford, and S.J. Miller, "An aggressive reduction scheme for the simple plant location problem," European Journal of Operational Research, vol. 234, no. 3, pp. 674-82, 2014.

[15] J. H. Lee, and T. Kwon, "GENDEP: Location-Aware Key Management for General Deployment of Wireless Sensor Networks," International Journal of Distributed Sensor Networks, 2014.

(C) Chen et al.; Licensee Bentham Open.

This is an open access articles licensed under the terms of the Creative Commons Attribution-Non-Commercial 4.0 International Public License (CC BY-NC 4.0) (https://creativecommons.org/licenses/by-nc/4.0/legalcode), which permits unrestricted, non-commercial use, distribution and reproduction in any medium, provided that the work is properly cited. 